Praveen Chahar, MD,FCARCSI

Center of Critical Care and Resuscitation,

Anesthesiology Institute, Cleveland Clinic
Donn Marciniak, MD

Center of Critical Care and Resuscitation,

Anesthesiology Institute, Cleveland Clinic

\title{
Cardiopulmonary resuscitation in COVID-19 patients
}

\section{Posted May 29, 2020}

\begin{abstract}
COVID-19 is extremely transmissible. Cardiopulmonary resuscitation (CPR) is associated with multiple aerosolgenerating procedures including chest compression, positive pressure ventilation, and airway manipulation. Healthcare providers who perform CPR are at high risk of contracting COVID-19. CPR in patients with suspected or proven COVID-19 must be performed without compromising the safety of healthcare providers. An overview of special considerations.
\end{abstract}

\section{BACKGROUND}

COVID-19 is extremely transmissible with a high morbidity and mortality. Cardiopulmonary resuscitation (CPR) is associated with multiple aerosolgenerating procedures including chest compression, positive pressure ventilation, and airway manipulation. ${ }^{1}$ Viral particles can remain suspended in air with a half-life of 1 hour. ${ }^{2}$ Healthcare providers performing $\mathrm{CPR}$ are thus at highest risk of contracting the disease due to working in close proximity to COVID-19 patients. ${ }^{3}$ CPR in patients with suspected or proven COVID-19 is aimed at achieving the return of spontaneous circulation without compromising the safety of caregivers.

Causes of cardiac arrest in COVID-19 patients include distributive or cardiogenic shock, hypoxia, and use of QT-prolonging drugs such as azithromycin and hydroxychloroquine. ${ }^{4,5}$ Cardiac arrest in patients with COVID-19 is associated with very poor outcomes. A study in Wuhan, China, reported that return of spontaneous circulation was achieved with CPR in only $13 \%$ of COVID-19 patients, with only

The statements and opinions expressed in COVID-19 Curbside Consults are based on experience and the available literature as of the date posted. While we try to regularly update this content, any offered recommendations cannot be substituted for the clinical judgment of clinicians caring for individual patients.

doi:10.3949/ccjm.87a.ccc040
$2.9 \%$ alive at 30 days. ${ }^{6}$ By comparison, a study from Beijing, China, reported a rate of return of spontaneous circulation of $35.5 \%$ and a hospital discharge rate of $9.1 \%$ in non-COVID-19 patients. ${ }^{?}$

\section{CONSIDERATIONS BEFORE RESUSCITATION}

The need for advance directives should be addressed with all patients with confirmed or suspected COVID-19 (or with the patient's proxy) on arrival at the hospital and revised with any changes in clinical condition. ${ }^{1}$ Patients with respiratory distress require a prompt decision to intubate to prevent peri-intubation cardiac arrest and cross-infection that may occur during emergency intubations.

Deteriorating patients should be preferentially transferred to negative-pressure rooms to minimize risk of exposure to providers during CPR. Mechanical compression devices for CPR should be kept in units housing patients with COVID-19. Caregivers assigned to a resuscitation team should have a bag with personal protective equipment (PPE) immediately available, with contents verified prior to start of shift.

In the event of cardiac arrest, all providers should don appropriate PPE before entering the room, even if this delays care. The number of caregivers should be minimized, and only caregivers with assigned roles should enter the room. Extra caregivers should be outside the room with a team member donned in PPE to help with medications and equipment if needed by the team. We recommend practicing a mock code tailored to considerations specific to COVID-19 patients.

\section{CPR AND COVID-19:}

\section{ADDITIONAL CONSIDERATIONS}

"Compression-only" (hands-only) CPR should be administered by the first available provider in appropriate PPE. A barrier device, cloth, or mask can be 
placed on the patient's mouth to minimize aerosol generation.

For shockable rhythms (ventricular tachycardia, ventricular fibrillation), defibrillator pads should be placed and shock applied as soon as a defibrillator is available. ${ }^{1}$ In the absence of reliable intravenous access, we recommend early intraosseous line placement. A mechanical compression device (LUCAS device, AutoPulse) should be used as soon as available. Passive oxygenation should be provided by a non-rebreathing mask covered by a surgical mask. Use of bag-mask ventilation and rescue breaths is discouraged. Placement of supraglottic device with a high-efficiency particulate air (HEPA) filter attached is preferred to ventilate the patient while a definitive airway is placed if absolutely necessary. The advanced cardiac life support (ACLS) protocol should be followed with early placement of an endotracheal tube. ${ }^{1}$

All ACLS medications are used in standard manner. The mechanical compression device should be used in continuous mode while preparing for intubation and chest compressions, and it should be paused during attempted intubation. Intubation should be performed by the most experienced provider, and a video laryngoscope is preferred to minimize intubation attempts. Use of a barrier device is encouraged while intubating to prevent cross-contamination. A HEPA filter should be attached as soon as the endotracheal tube is placed, before the first delivered breath. Auscultation to confirm endotracheal tube placement is discouraged, but tube placement can be confirmed with visualization of the endotracheal tube through the vocal cords, end-tidal $\mathrm{CO}_{2}$ capnometry or capnography, bilateral lung-sliding on chest ultrasonography, and chest radiography.

Bronchoscopic intubation is not recommended due to the risk of aerosol generation; bronchoscopy should be performed only if absolutely necessary. Disposable bronchoscopes are highly recommended.

The patient should be connected to a ventilator with a HEPA filter as soon as possible, and we recommend two-person verification of proper filter placement. Disconnections in the breathing circuit should be avoided. If intubation is done at the time of cardiac arrest, the patient should be maintained on a ventilator with a HEPA filter. The mode of ventilation should be changed to asynchronous with pressure control with inspiratory pressure sufficient for chest rise or tidal volume of 6 to $8 \mathrm{~mL} / \mathrm{kg}$. The fraction of inspired oxygen should be set to $100 \%$, with positive end-expiratory pressure set to zero. The respiratory rate should be set to 10 breaths per minute. The trig- ger on the ventilator should be switched off to prevent auto-triggering with chest compressions. Ventilator settings can be changed as appropriate once return of spontaneous circulation is achieved.

\section{CPR WITH PATIENT IN PRONE POSITION}

CPR in prone position in COVID-19 patients with advanced airway in place is controversial and is not practiced widely. The American Heart Association (AHA) recommendation is to avoid turning patients supine if advanced airway is in place, due to the risk of circuit disconnection and aerosolization. The AHA suggests placing pads in anterior-posterior position and providing CPR with hands in standard position over the T7 to T10 vertebral bodies. The efficacy of CPR in prone position is not completely known and is performed rarely, balancing the futility of treatment vs the chance of return of spontaneous circulation.

\section{POST-ARREST CONSIDERATIONS}

The mortality rate in critically ill patients with COVID-19 is high and increases with comorbidities. CPR is a resource-consuming effort. The patient's comorbidities and condition at the time of cardiac arrest need to be taken into consideration to decide the appropriateness of continuing resuscitation.

There is insufficient evidence to support the use of extracorporeal membrane oxygenation in COVID19 patients during cardiac arrest. These patients are poor candidates for the procedure, and it is not recommended.

Care after cardiac arrest in COVID-19 patients is similar to that for other patients, with a focus on minimizing cross-infection and appropriate triaging of resources. While transporting these patients, it is important to follow infection-control policies of the institute, and any non-disposable equipment should be cleaned according to infection-control policies. Doffing and hand hygiene should be performed using a buddy system.

\section{REFERENCES}

1. Edelson DP, Sasson C, Chan PS, et al. Interim guidance for basic and advanced life support in adults, children, and neonates with suspected or confirmed COVID-19: from the Emergency Cardiovascular Care Committee and Get With the Guidelines-Resuscitation Adult and Pediatric Task Forces of the American Heart Association in collaboration with the American Academy of Pediatrics, American Association for Respiratory Care, American College of Emergency Physicians, The Society of Critical Care Anesthesiologists, and American Society of Anesthesiologists: supporting organizations: American Association of Critical Care Nurses and National EMS Physicians. Circulation 2020; April 9. doi:10.1161/CIRCULATIONAHA.120.047463

2. van Doremalen N, Bushmaker T, Morris DH, et al. Aerosol and sur- 
face stability of SARS-CoV-2 as compared with SARS-CoV-1. N Engl J Med 2020; 382(16):1564-1567. doi:10.1056/NEJMc2004973

3. Gamio L. The workers who face the greatest coronavirus risk. New York Times 2020.

4. Guo T, Fan Y, Chen M, et al. Cardiovascular implications of fatal outcomes of patients with coronavirus disease 2019 (COVID-19). JAMA Cardiol 2020; Mar 27. doi:10.1001/jamacardio.2020.1017

5. Shao F, Xu S, Ma X, et al. In-hospital cardiac arrest outcomes among patients with COVID-19 pneumonia in Wuhan, China. Resuscitation 2020; 151:18-23. doi:10.1016/j.resuscitation.2020.04.005

6. Shao F, Li CS, Liang LR, et al. Incidence and outcome of adult in-hospital cardiac arrest in Beijing, China. Resuscitation 2016; 102:51-56. doi:10.1016/j.resuscitation.2016.02.002

7. Mazer SP, Weisfeldt M, Bai D, et al. Reverse CPR: a pilot study of CPR in the prone position. Resuscitation 2003; 57(3):279-285. doi:10.1016/s0300-9572(03)00037-6 